\title{
Editorial
}

\section{Recent Developments on Time-Delay Neural Networks}

\author{
Zheng-Guang Wu, ${ }^{1}$ Yun Chen, ${ }^{2}$ Xusheng Lei, ${ }^{3}$ Kun Liu, ${ }^{4}$ and Hui Zhang ${ }^{5}$ \\ ${ }^{1}$ Zhejiang University, Hangzhou 310027, China \\ ${ }^{2}$ Hangzhou Dianzi University, Hangzhou 310018, China \\ ${ }^{3}$ Beijing University of Aeronautics and Astronautics, Beijing 100191, China \\ ${ }^{4}$ KTH Royal Institute of Technology, 10044 Stockholm, Sweden \\ ${ }^{5}$ The Ohio State University, Columbus, OH 43210, USA \\ Correspondence should be addressed to Zheng-Guang Wu; nashwzhg@126.com
}

Received 6 May 2015; Accepted 6 May 2015

Copyright (C) 2015 Zheng-Guang Wu et al. This is an open access article distributed under the Creative Commons Attribution License, which permits unrestricted use, distribution, and reproduction in any medium, provided the original work is properly cited.

Over the past several decades, an amount of attention has been devoted to neural networks, which have wide applications in many areas, such as signal processing, pattern recognition, and combinatorial optimization. On the other hand, time delays occur in electronic implementation of analog neural networks because of the transmission of signal and the finite switching speed of amplifiers and can lead to the instability and poor performance of systems; thus neural networks with time delay have also been studied extensively because numerous interesting results have been obtained over the last decade, including stability analysis, state estimation, and passivity.

In this special issue, we have accepted seven papers. The paper titled "New Pinning Synchronization of Complex Networks with Time-Varying Coupling Strength, NonDelayed and Delayed Coupling" by G. Wang et al. studies the pinning synchronization problem for a class of complex networks by a stochastic viewpoint, in which both time-varying coupling strength and nondelayed and delayed coupling are included. The paper titled "A Novel Model of Conforming Delaunay Triangulation for Sensor Network Configuration" by Y. Ma et al. proposes a new method for solving Delaunay Triangulation problem, which is called endpoint triangle's circumcircle model (ETCM). The paper titled "Multimodel Modeling and Predictive Control for DirectDrive Wind Turbine with Permanent Magnet Synchronous Generator" by L. Wang et al. establishes a method based on multimodel modeling and predictive control is proposed for the optimal operation of direct-drive wind turbine with permanent magnet synchronous generator. The paper titled "Independent Component Analysis Based on Information Bottleneck" by Q. Ke et al. provides the equivalence of two algorithms of independent component analysis (ICA) based on the information bottleneck (IB). In "Nonlinear Time-Delay Suspension Adaptive Neural Network Active Control" by Y. Zhu and S. Zhu, a quarter-vehicle magnetorheological active suspension nonlinear model with time delay is established, and an adaptive neural network structure for magnetorheological active suspension is presented. The paper titled "An Adaptive Regulator for Space Teleoperation System in Task Space" by C. Ge et al. studies the problem of the gravity information for bilateral teleoperation. The paper titled "Robust Stabilization of Linear Switching Systems with both Input and Communication Delays" by L. Jia and Z. Sheng considers the stabilization control of a class of linear switching systems with time delays in both the input and the communication channels.

\section{Acknowledgment}

We would like to express our great appreciation to all the authors of this special issue and all the reviewers.

$$
\begin{array}{r}
\text { Zheng-Guang Wu } \\
\text { Yun Chen } \\
\text { Xusheng Lei } \\
\text { Kun Liu } \\
\text { Hui Zhang }
\end{array}
$$




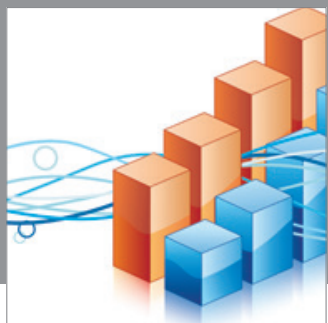

Advances in

Operations Research

mansans

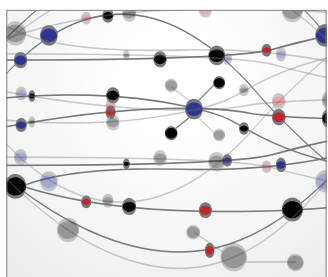

The Scientific World Journal
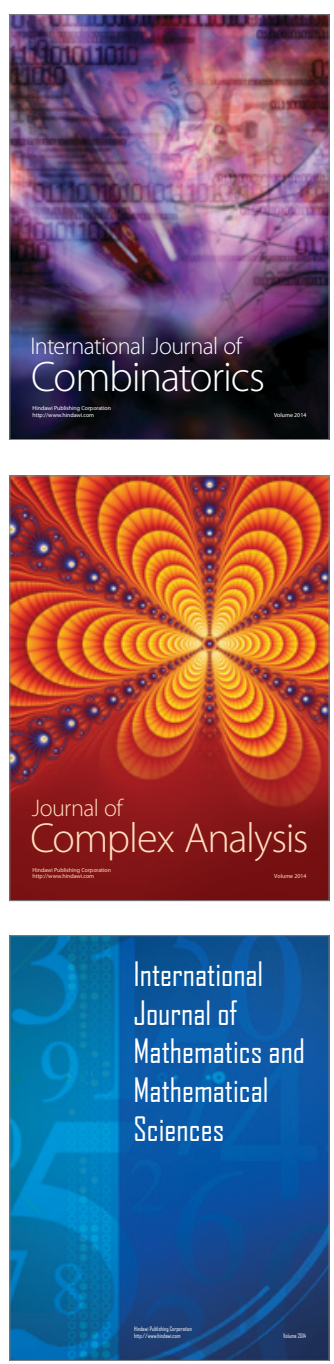
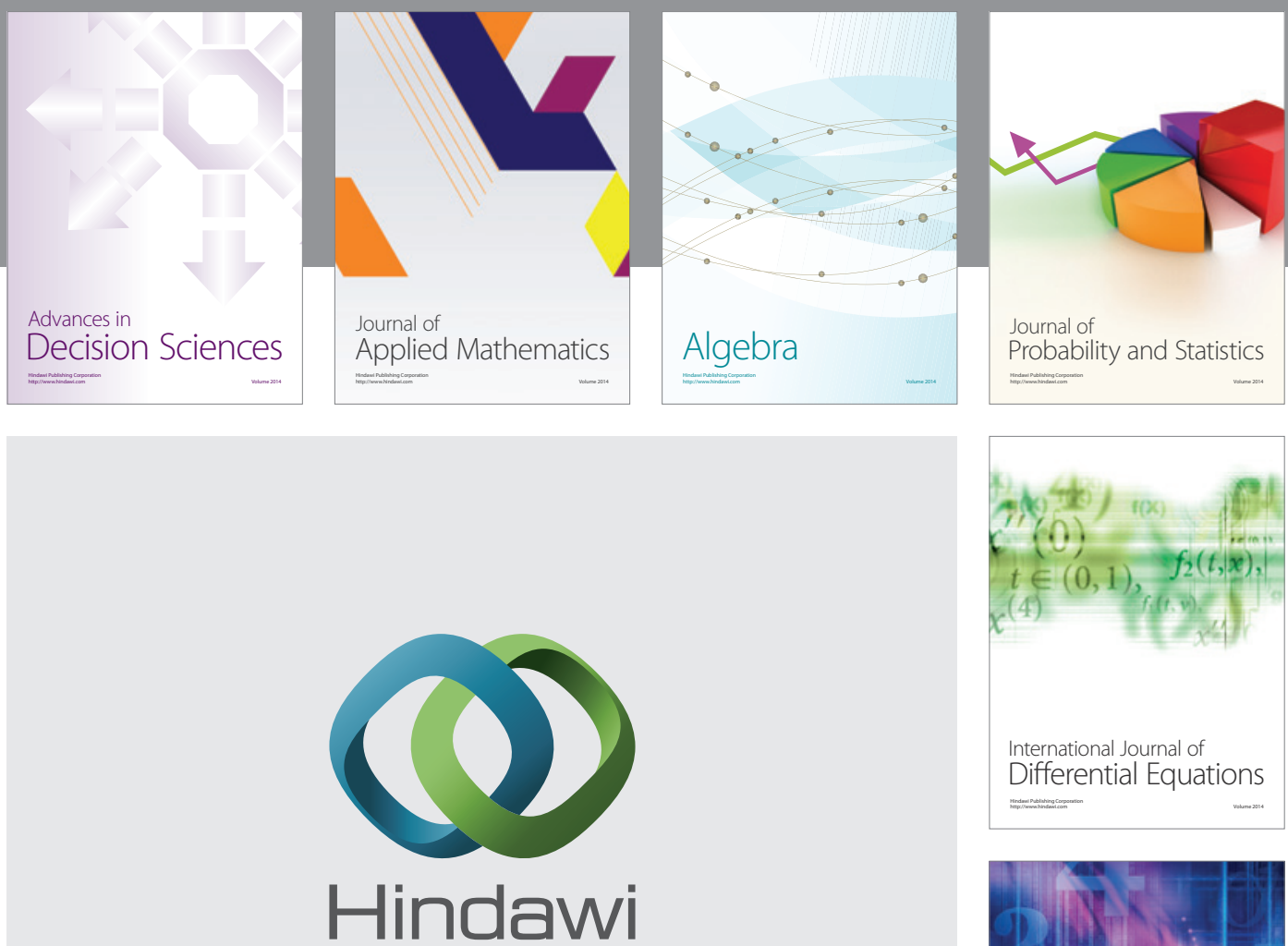

Submit your manuscripts at http://www.hindawi.com
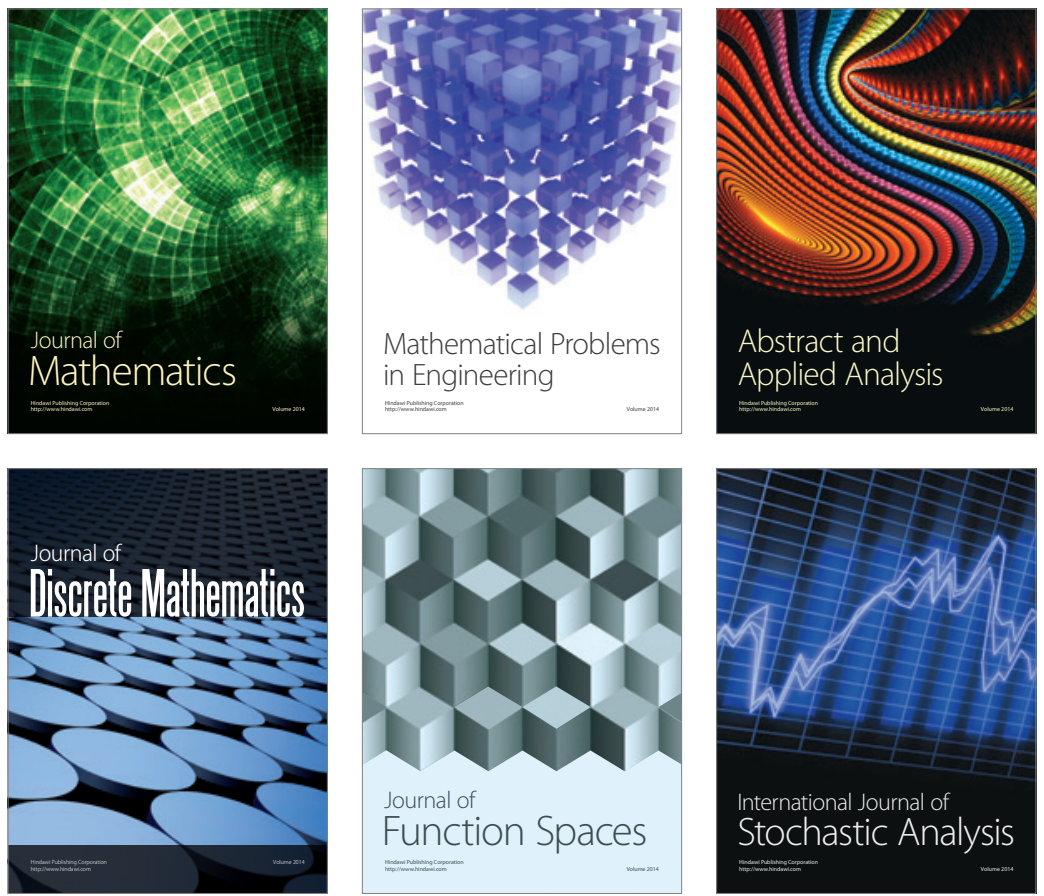

Journal of

Function Spaces

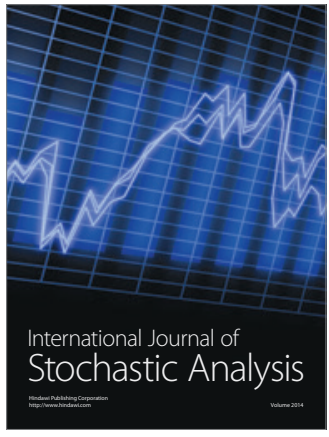

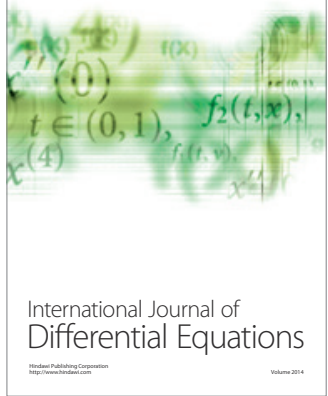
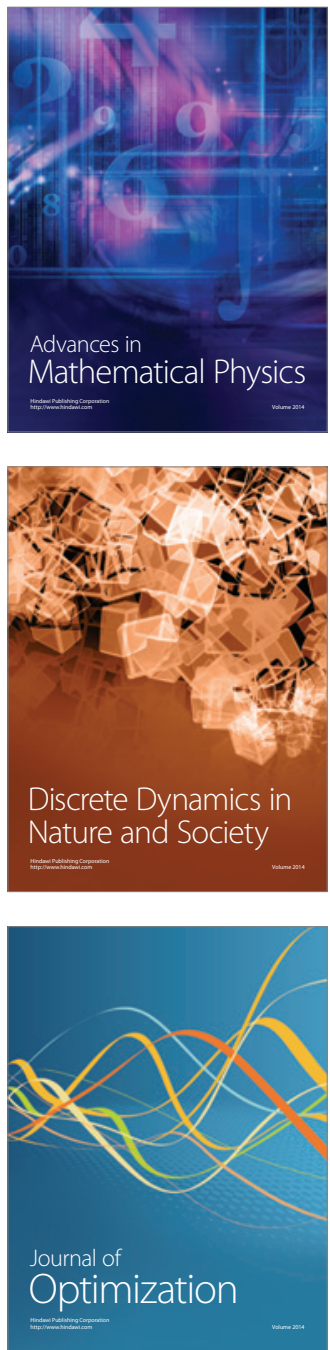